\title{
A performance analysis of general packet radio service (GPRS) and narrowband internet of things (NB-loT) in Indonesia
}

\author{
I Wayan Krisnhadi Bima W'ㄹ, Vera Suryani ${ }^{\star 2}$, Aulia Arif Wardhana ${ }^{3}$ \\ Telkom University, Bandung ${ }^{1,2,3}$
}

\author{
Article Info \\ Keywords: \\ Internet of Things, General Packet Radio \\ Service, Narrowband-IoT, Packet Loss \\ Article history: \\ Received 30 September 2019 \\ Revised 05 November 2019 \\ Accepted 17 January 2020 \\ Published 06 February 2020

\section{Cite:} \\ Bima, I., Suryani, V., \& Wardana, A. (2020). A \\ Performance Analysis of General Packet \\ Radio Service (GPRS) and Narrowband \\ Internet of Things (NB-IoT) in \\ Indonesia. Kinetik: Game Technology, \\ Information System, Computer Network \\ Computing, Electronics, and Control, 5(1). \\ doi:https://doi.org/10.22219/kinetik.v5i1.947 \\ ${ }^{*}$ Corresponding author. \\ Vera Suryani \\ E-mail address: \\ verasuryani@telkomuniversity.ac.id
}

\begin{abstract}
Internet of Things (loT) refers to a concept connecting any devices onto the internet. The loT devices are able to not only use a service or server to be control at distance but also to do computation. loT has been applied in many fields such as smart city, industries and logistics. The sending of loT data can use the existing GSM networks such as GPRS. However, GPRS is not dedicated particularly for the transmission of loT data in consideration to its weaknesses in terms of coverage and power efficiency. To increase the performance of the transmission of loT data, Narrowband-loT (NB-loT), one alternative to replace GPRS, is offered for its excellences in coverage and power. This paper aims to compare the GPRS and NB-loT technology for the transmission of IoT data, specifically in Bandung region, Indonesia. Considering that NB-loT is a new technology, a preliminary feasibility study of this technology is needed, especially in Indonesia. This paper is the first paper to discuss the performance analysis of NB-loT technology in Indonesia in terms of network performances. This research results can be useful for other further researches in NB-loT area. The results obtained showed that the packet loss from clients for the GPRS network was at $68 \%$, while the one for NB-loT was at $44 \%$. Moreover, NB-loT technology was found excellent in terms of battery saving compared to GPRS for the transmission of loT data. This then showed that NB-IoT was found more suitable for transmitting the loT data compared to GPRS.
\end{abstract}

\section{Introduction}

Internet of Things (IoT) is a concept to connect any devices or appliances onto internet. An appliance will be seen as "Internet of Thing" if it is able to do a processing on the embedded processors or microcontrollers, communicate, and be controlled or use a server or service on Internet [1][2]. The structure of loT consists of three components: hardware (sensor, actuator, embedded system, and communication), middleware (data processing from loT, analytical) and presentation (visualization and interpretation) to users [2][3]. Based upon the architecture of loT in [4], it requires an internet connection to connect things. The internet connection on loT can use the networking technology that has been available in the today telecommunication network such as GSM technology. In essence, any network connections can be used for loT data as long as their structure is compatible with the characteristics in loT data.

One of GSM technology that can be used for the transmission of loT data is General Packet Radio Service (GPRS). This technology, nevertheless, still has two weaknesses in its implementation regarding the use of high power and low coverage area. A solution offered is the network of Narrowband-loT (NB-loT), a protocol proposed by 3 GPP to replace GPRS protocol [5]. NB-IoT can use a little part of LTE network [6] and has a gain of 20dB in an indoor environment (such as in a tunnel) in comparison to GPRS. In addition, NB-loT is more power saving for being designed to the low power devices, off-the-grid / dependent upon the battery and has a network performance resembling GPRS $[5]$.

The use of Narrowband-loT has widely been applied on the smart metering or smart grid application [7][8][9][10][11]. Other Narrowband-loT-based applications include smart parking [12][13], smart cities [14][15][16][17][18][19][20][21]. Any applications that can be employed using NB-loT network need to adjust with the regulation of its deployment area. In Indonesia, not all base-stations support the NB-loT network. To make loT application can be used well in the NB-loT network, then there is a need for a study on the properness in the usage of the network for the loT data transmission.

There are some performance evaluations from other works that analyze the performance of NB-loT. The research [22] compared the performance and the power characteristic between 4G LTE and GPRS network. The research used smartphone mobile networks for its testing environment. The result for TCP test using parameter of Round Trip Time (RTT) from the LTE technology resulted in median value around $69.5 \mathrm{~ms}$, from the raw data ranging from $25 \mathrm{~ms}$ to 120

Cite: Bima, I., Suryani, V., \& Wardana, A. (2020). A Performance Analysis of General Packet Radio Service (GPRS) and Narrowband Internet of Things (NB-loT) in Indonesia. Kinetik: Game Technology, Information System, Computer Network, Computing, Electronics, and Control, 5(1). doi:https://doi.org/10.22219/kinetik.v5i1.947 
$\mathrm{ms}$. Meanwhile, from the $3 \mathrm{G}$ technology, the result of RTT value ranging from $200 \mathrm{~ms}$ to $400 \mathrm{~ms}$, and the median value was around $110 \mathrm{~ms}$.

The average of LTE throughput for uplink and downlink are $12.74 \mathrm{Mbps}$ and $5.64 \mathrm{Mbps}$. Although LTE has capability of high throughput, it scales with power consumption. For 5 Mbps downlink, LTE consumes power around $1700 \mathrm{~mW}$, for 5 Mbps uplink, it consumes around $3400 \mathrm{~mW}$, twice the consumption power of downlink. Base power consumption of LTE is $1060 \mathrm{~mW}$. Average 3G throughput are $2.5 \mathrm{Mbps}$ and $\leq 1$ Mbps for downlink and uplink respectively. Power consumption for 500 Kbps downlink, is $850 \mathrm{~mW}$ and $1250 \mathrm{~mW}$ for $500 \mathrm{Kbps}$ uplink. Base power consumption of $3 \mathrm{G}$ is $601 \mathrm{~mW}$.

\begin{tabular}{ccccc}
\multicolumn{5}{c}{ Table 1. Energy Usage Compared to Data Size in LTE and 3G } \\
\hline $\begin{array}{c}\text { Data Size } \\
\text { (B) }\end{array}$ & $\begin{array}{c}\text { LTE uplink } \\
(\mu \mathrm{J} / \mathrm{bit})\end{array}$ & $\begin{array}{c}\text { LTE downlink } \\
(\mu \mathrm{J} / \mathrm{bit})\end{array}$ & $\begin{array}{c}\text { 3G uplink } \\
(\mu \mathrm{J} / \mathrm{bit})\end{array}$ & $\begin{array}{c}\text { 3G downlink } \\
(\mu \mathrm{J} / \mathrm{bit})\end{array}$ \\
\hline 10000 & $\sim 10$ & $\sim 5.9$ & $\sim 13.1$ & $\sim 8.1$ \\
100000 & $\sim 3$ & $\sim 1.5$ & $\sim 7$ & $\sim 5.8$ \\
1000000 & $\sim 1.8$ & $<0.5$ & $\sim 6.5$ & $\sim 5.5$ \\
10000000 & $<1.5$ & $<0.1$ & $\sim 6.4$ & $\sim 4.8$ \\
\hline
\end{tabular}

Table 1 depicts that LTE is not suitable for transferring small data and staying in an idle state. The advantage of $3 G$ and $4 G$ depends on the application. $4 G$ is wasted in power usage, but its capability of high throughput is suitable for high definition urban security camera that is always connected to main power. $3 G$ is may suitable for remote application that is powered by a battery that is intermittently charged from other power sources (such as solar panel) and needs adequate throughput to do its function properly.

The other study [20][23] analyze power consumption and battery life from NB-loT. The goal of the study is to measure power usage when transmitting the signal. The study also estimates the battery life when the device sends a signal using NB-loT. The result is NB-loT commercial device consumes $716 \mathrm{~mW}$ to transmit a $23 \mathrm{dBm}$ signal. Other studies [24] focus on signal coverage analysis from Sigfox, LoRa, GPRS, and NB-loT. The testing purpose was to measure the failure rate from uplink and downlink from the signal transmission. The result from the study showed that NB-IoT had a failure rate below $5 \%$ for downlink and uplink connectivity in indoor and outdoor coverage. The last, a study presented in [25] compared signal coverage between LTE-M and NB-IoT in the rural area. The goal was to measure the coverage signal in an indoor and outdoor location. The result was NB-loT could coverage $95 \%$ use in a deep indoor location.

Based on previous studies, no other studies focused on performance analysis of NB-loT and GPRS in data transmission based on the network condition. This paper presents a performance test on the properness of the usage of NB-loT network in Indonesia - particularly in Bandung. This test can be seen in terms of latency, throughput, packet loss, and power usage.

The paper is organized as follows: Section 2 describes the research method, Section 3 describes the simulation, result, and discussion of performance evaluation and Section 4 explains the conclusion and future works.

\section{Research Method}

In this research, the performance test from GPRS and NB-loT was conducted to compare the network in transmitting the data from device onto cloud server. GPRS and NB-loT are a mobile network requiring Base Transmission Station (BTS) from a cellular operator for communication. The mobile network applies a mechanism of radio wave to build a network in transmitting the data. The radio wave is used to provide the service of mobility and capacity to cover a wide area [5][26][27]. The longer the transmitted radio wave, the larger the delay. Delay occurs when the transmission can be influential in the throughput of a network. Therefore, the measurement of performance towards the throughput is very important as the parameter of performance in mobile network [26][28].

Radio wave also has the uplink or downlink when transmitting a data. The transmission of uplink occurs between device and BTS tower; on the other hand, the downlink transmission occurs between BTS and device [5][26]. Each transmission of uplink and downlink also brings an effect on the performance of data transmission. The Round Time Trip (RTT) of the data sent from a device to a BTS and then forwarded to the server and returning to the device through BTS is the parameter of performance of uplink and downlink of a mobile network [26][29].

In addition, the power usage in device brings an effect on the transmission of uplink and downlink on mobile network. The radio wave transmitted by the device is also determined by the electrical power existing in the device. The longer the transmission of radio wave in the device, the larger the power required [20][23].

Based on the justification of the measurement methods above, then the performance test was conducted to achieve the following Key Performance Indicators (KPI):

1. The measurement of throughput for the performance of the network in data transmission.

2. The RTT measurement to measure the time between the transmission and receipt of data package.

(c) 2020 The Authors. Published by Universitas Muhammadiyah Malang

This is an open access article under the CC BY SA license. (https://creativecommons.org/licenses/by-sa/4.0/) 
3. The measurement of power consumption to measure the power use when the device transmits the data.

Once KPI had been determined from the measurement to be done, then a test scenario was made. In this research. It began by explaining the coverage area from the network of NB-loT and GPRS existing in Bandung area, Indonesia. The information about the coverage was used to find out to what extent the coverage area from the network of NB-IoT and GPRS to make it possible to be used as the reference of the mobility of the test. Subsequently, two scenarios of test on the network performance of the analysis were defined consisting of test towards the throughput and RTT from the data transmission. To figure out the power consumption of GPRS and NB-loT when transmitting the data, then the test scenario of the power consumption was made.

\subsection{Coverage Area}

Prior to measure the performance of mobile networks on GPRS and NB-loT it was necessary to know the coverage area of the two technologies, as seen in Table 2. The test was conducted in the campus area of Telkom University, Bandung, Indonesia. The first thing to do to find out the coverage area was to measure the signal strength in the test area. Signal strength on mobile networks is commonly measured by using the units of dBm (decibelmilliwatts). Signal strength is the representation of how much signal is received from cellular networks (downlink mode) [24][25].

Table 2. Signal Strength Condition Standard from 2G and 3G Network

\begin{tabular}{|c|c|c|}
\hline RSSI & Signal Strength & Description \\
\hline$\geq-70 \mathrm{dBm}$ & Excellent & Strong signal with maximum data speeds \\
\hline$-70 \mathrm{dBm}$ to $-85 \mathrm{dBm}$ & Good & Strong signal with good data speeds \\
\hline$-86 \mathrm{dBm}$ to $-100 \mathrm{dBm}$ & Fair & $\begin{array}{l}\text { Fair but useful, fast and reliable data speeds } \\
\text { may be attained, but marginal data with drop- } \\
\text { outs is possible }\end{array}$ \\
\hline \multirow{2}{*}{$\begin{array}{c}<-100 \mathrm{dBm} \\
-110 \mathrm{dBm}\end{array}$} & Poor & Performance will drop drastically \\
\hline & No signal & Disconnection \\
\hline
\end{tabular}

There are two scenarios of measurement in the network measurement to be done. The first measurement was related to the throughput of GPRS and NB-loT networks. In the tests conducted, measurement of throughput was calculated using Equation 1.

$$
T=\frac{\sum n K b i t}{S}
$$

Throughput refers to the actual bandwidth measured by time units and certain network conditions to send data with a certain size. Based on Equation 1, the throughput test was carried out by sending a certain amount of data carried out over a certain period of time. The transmission was made from the device to the server via GPRS and NB-loT networks. The second measurement was related to RTT from GPRS and NB-loT networks. In the tests carried out, RTT measurements were calculated using Equation 2.

$$
R T T=t A-t D
$$

From Equation 2, the RTT test was to measure the time range between the time of data transmission and the time of arrival of the transmitted data. Test was to calculate the RTT of data sent from the device to the server and returned to the device.

\subsection{Power Consumption Analysis}

In the test of power consumption, two testing categories of testing were analysed. First, it was regarding the overall power usage on the device when using GPRS and NB-loT networks. Second, the device utility was analysed when transmitting data on GPRS and NB-loT networks. In the utility test, the measurement of power consumption estimation on the battery usage when transmitting and using the GPRS and NB-loT was also done. The overall measurement of power usage on GPRS and NB-IoT networks used the Joule unit. In the measurement of overall power consumption performed on GPRS and NB-loT was calculated using Equation 3.

$$
J=\frac{W}{S}
$$

Cite: Bima, I., Suryani, V., \& Wardana, A. (2020). A Performance Analysis of General Packet Radio Service (GPRS) and Narrowband Internet of Things (NB-loT) in Indonesia. Kinetik: Game Technology, Information System, Computer Network, Computing, Electronics, and Control, 5(1). doi:https://doi.org/10.22219/kinetik.v5i1.947 
Testing using Equation 3 measured the overall power usage of the device. The overall measurement of power consumption included power usage when using GPRS / NB-loT networks and reading data from sensors.

The measurements of GPRS and NB-IoT usage were conducted using two scenarios. First, power consumption was measured when the device transmitted the data. Second, the time estimation towards the power in the battery was measured. Those two measurement scenarios were expected to illustrate the power usage used by devices with GPRS or NB-IoT mobile networks. In the first utility measurement, the power consumption was measured when the device transmitted the data. The measurement was conducted on GPRS and NB-IoT and calculated using Equation 4.

$$
P C=\frac{T}{T p}
$$

From Equation 4, the power consumption was measured based upon the size of the throughput generated at one time data transmission. The size of the throughput was compared to the transmit power when transmitting or receiving data. The measurement of power consumption when the device transmitted the data was conducted on GPRS and NBIoT and calculated using Equation 5.

$$
\text { Estimated Time }=\frac{\frac{\text { Transaction } * \text { data length }(\text { byte })}{\text { Transaction interval }(s)} * \text { Transmit power }(W / \text { byte })}{\text { Battery capacity }(W s)}
$$

Based on Equation 5, the measurement would be focused on power usage when transmitting the data only using the network of GPRS or NB-IoT. The measurement was initially done to the data transaction to be transmitted. Furthermore, the measurement was combined with the amount of power consumption in each data transaction. This was then continued by comparing the battery power used to turn on the device.

\section{Results and Discussion}

This section explains the environment used for testing in this research. The topology from the network used for testing is describing in point 3.1.

\subsection{Feasibility Design}

Narrowband-IoT employs traditional GSM topology, Figure 1 is the simplified version of the network topology.

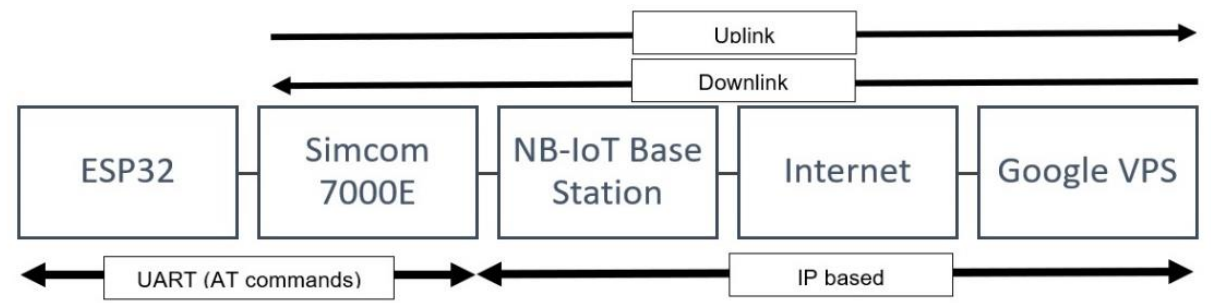

Figure 1. Topology Diagram

The detail from the testing scenario and environment is describing in point 3.1.1 and 3.1.2. The pseudocode from the network performance equation that used for testing is described in point 3.1.3.

\subsubsection{Test Scenario and Parameters}

These lists are test parameters, devices specifications, and location.

a) Devices and equipment

a. ESP32 WROOM (4 MB flash, 512 KB RAM)

b. Waveshare SIMcom 7000E module

Specification and capabilities [30] :

- Frequency bands: GSM (900 Mhz / 1800 Mhz), LTE-M1, LTE-NB1

- GNSS for geolocation data

- Operating voltage: 3.3 volts -4.03 volts (module built in with 3.3 volt to 5 volt regulator)

- Transmit power:

$$
\begin{array}{ll}
\circ & \text { GPRS: } 2 \mathrm{~W} \text { (EGSM 900), } 1 \mathrm{~W} \text { (DCS1800) } \\
\circ & \text { EGDE: } 0.5 \mathrm{~W} \text { (EGSM 900), } 0.4 \mathrm{~W} \text { (DCS1800) } \\
\circ & \text { LTE: } 0.25 \mathrm{~W}
\end{array}
$$

(C) 2020 The Authors. Published by Universitas Muhammadiyah Malang

This is an open access article under the CC BY SA license. (https://creativecommons.org/licenses/by-sa/4.0/) 
- Data transmission speed:

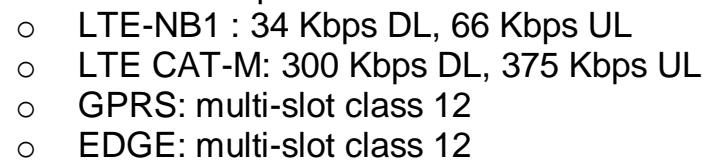

- UART interface (115200 bps)

- USB interface

c. Laptop/Tablet as debugging/terminal display

d. SANFIX DM-888 multimeter for power usage measurement.

e. Google cloud VPS as test server

- Runs ASP.net core 2.0 web API server

- Python 3.0 for UDP server

- Located in Singapore

b) Test location and signal coverage
a. Jalan Telekomunikasi, Universitas Telkom, Bojongsoang, Bandung
b. GPRS signal strength: $-71 \mathrm{dBm}$
c. NB-loT signal strength $-87 \mathrm{dBm}$

\subsubsection{Data Payload:}
a. TCP using HTTP:
- Upload: $5 \mathrm{~KB}$
- Download: $10 \mathrm{~KB}$
b. UDP:
- Time out: 60 seconds
- Send: $1 \mathrm{~KB}$
- Receive: $1 \mathrm{~KB}$ (echo)
- Time out: 10 seconds
c. Test iteration: 50 times
d. eDRX configuration for NB-loT: $5.52 \mathrm{~s}\left(1^{\text {st }}\right.$ cycle $)$

\subsubsection{Pseudocode}

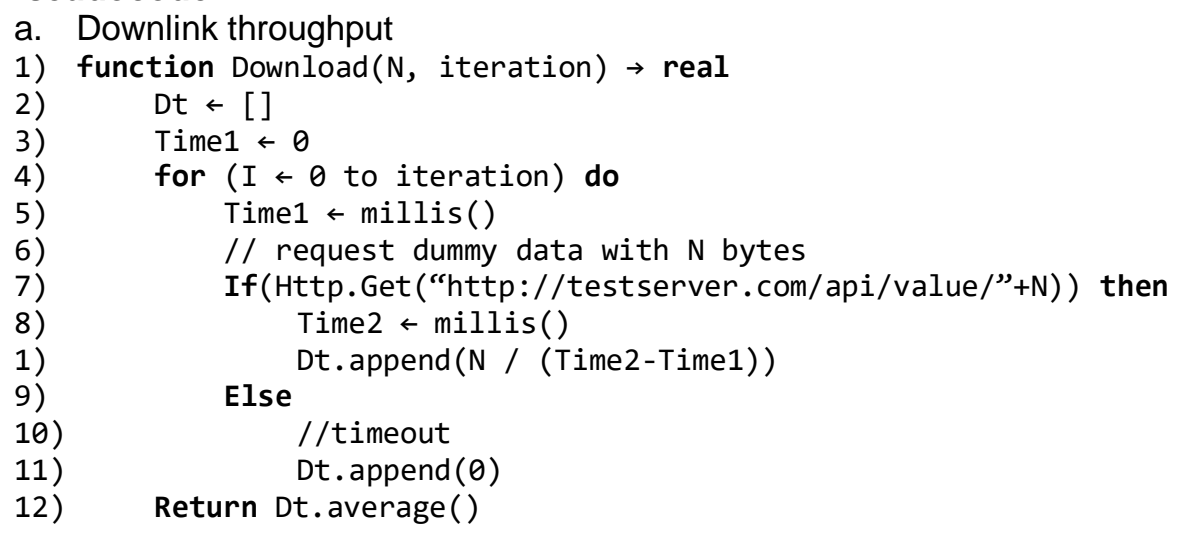

b. Uplink throughput

1) function UploadTest( $N$, iteration) $\rightarrow$ real

2) Dt $\leftarrow$ []

3) // create dummy array, fill it with As

4) dummyData $\leftarrow$ new $\operatorname{char}[\mathrm{N}]$

5) memset (dummyData, ' $A$ ', $N$ )

6) Time1 $\leftarrow 0$

7) for ( $I \leftarrow 0$ to iteration) do

8) Time1 $\leftarrow$ millis()

9) //send that array, we assume that server only sent empty response,

10) //because we're only measuring uplink throughput

11) If(Http.Post("http://testserver.com/api/value/"+N, dummyData, length)) then

12) Time $2 \leftarrow \operatorname{millis}()$ 

13)
14)
15)
16)
17)

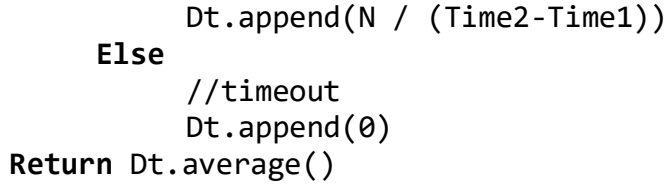

\subsection{Performance profiling}

Table 3 depicted the result of RTT TCP benchmarking for GPRS and NB-IoT. This research used TCP protocol in the transport layer and used the HTTP protocol in the application layer. Table 3 measure HTTP Round Trip Time by uploading $1 \mathrm{~KB}$ upload payload and $10 \mathrm{~KB}$ download payload. Round Trip Time did not include timed out of a transaction. Maximum transaction time was 60 seconds; otherwise it was counted as "timed out" or "fail". For each upload and download did as much 50 times. The fail attempt is the number of fails to upload a payload (received an empty response) and download a payload (sent empty request).

Table 3. RTT TCP Benchmark for GPRS and NB-IOT

\begin{tabular}{cccc}
\hline Network & Round Trip Time & $\begin{array}{c}\text { Fail Attempt } \\
\text { (Upload) }\end{array}$ & $\begin{array}{c}\text { Fail Attempt } \\
\text { (Download) }\end{array}$ \\
\hline GSM/GPRS & $17422.875 \mathrm{~ms}$ & 13 & 9 \\
NB-loT & $8007.96 \mathrm{~ms}$ & 7 & 17 \\
\hline
\end{tabular}

In Table 4, UDP Round Trip Time was measured by sending a 1 KB UDP echo message to reach the server and back in a certain time period. Round Trip Time did not include timed out transaction. Maximum time to receive echo message was 10 seconds; otherwise it is counted as "failed." Fail Attempt was represented the number of UDP packet that did not reach the server or client.

Table 4. RTT UDP Benchmark for GPRS and NB-IOT

\begin{tabular}{cccc}
\hline Network & Round Trip Time & $\begin{array}{c}\text { Fail Attempt (Payload did } \\
\text { not reach server) }\end{array}$ & $\begin{array}{c}\text { Fail Attempt (Echo payload } \\
\text { did not reach client) }\end{array}$ \\
\hline GSM/GPRS & $1531.814 \mathrm{~ms}$ & $1 / 50$ & $33 / 50$ \\
NB-loT & $1434.091 \mathrm{~ms}$ & $0 / 50$ & $28 / 50$ \\
\hline
\end{tabular}

In Table 5, throughput from HTTP was measured by uploading $1 \mathrm{~KB}$ payload and downloading $10 \mathrm{~KB}$ payload in a certain period. Based on the experiment, the average throughput for uploading from GPRS and NB-loT had similar result. Furthermore, parameter of average download from NB-loT throughput was smaller than GPRS, and the value was not too far adrift with the throughput when uploaded.

Table 5. Throughput HTTP Benchmark for GPRS and NB-IoT

\begin{tabular}{ccc}
\hline Network & Average Upload & Average Download \\
\hline GSM/GPRS & $347.3 \mathrm{~B} / \mathrm{s}(2.776 \mathrm{Kbit} / \mathrm{s})$ & $2593.280 \mathrm{~B} / \mathrm{s}(20 \mathrm{Kbit} / \mathrm{s})$ \\
NB-loT & $350.760 \mathrm{~B} / \mathrm{s}(2.806 \mathrm{Kbit} / \mathrm{s})$ & $348.839 \mathrm{~B} / \mathrm{s}(2.7907 \mathrm{Kbit} / \mathrm{s})$ \\
\hline
\end{tabular}

From the HTTP test, it was concluded that NB-IOT has smaller RTT compared to GPRS. NB-IoT was also not suitable to download big files (in this case, downloading more than $10 \mathrm{~KB}$ data). In UDP test, NB-loT has marginally smaller RTT than GPRS but having better reliability.

Meanwhile, the energy usage for both GPRS and NB-IOT was measured in the operating voltage of 3.3 volts. The device sent $50 \mathrm{~KB}$ (1 KB each) UDP packets during the transmit power test. Transmit power was also measured within 20 seconds timeframe. This benchmark measured from modem power consumption. The result is depicted in Table 6.

Table 6. Energy Usage of GPRS and NB-IoT

\begin{tabular}{ccccc}
\hline Network & Start up & Idle \& Connected & Idle & Transmit \\
\hline GSM/GPRS & $7 \mathrm{~J}(0.35 \mathrm{~W})$ & $4.7 \mathrm{~J}(0.235 \mathrm{~W})$ & $6.15 \mathrm{~J}(0.307 \mathrm{~W})$ & $14.75 \mathrm{~J}(0.737 \mathrm{~W})$ \\
NB-loT & $8.25 \mathrm{~J}(0.412 \mathrm{~W})$ & $5.1 \mathrm{~J}(0.225 \mathrm{~W})$ & $5.05 \mathrm{~J}(0.252 \mathrm{~W})$ & $8.1 \mathrm{~J}(0.405 \mathrm{~W})$ \\
\hline
\end{tabular}

Small power consumption improves the device battery life. As seen in Figure 2, the power consumption of transmitting power from NB-loT was smaller than GPRS in the uplink mode. But the power consumption of transmitting power from NB-loT was bigger than GPRS in the downlink mode, as seen in Figure 3.

(c) 2020 The Authors. Published by Universitas Muhammadiyah Malang

This is an open access article under the CC BY SA license. (https://creativecommons.org/licenses/by-sa/4.0/) 


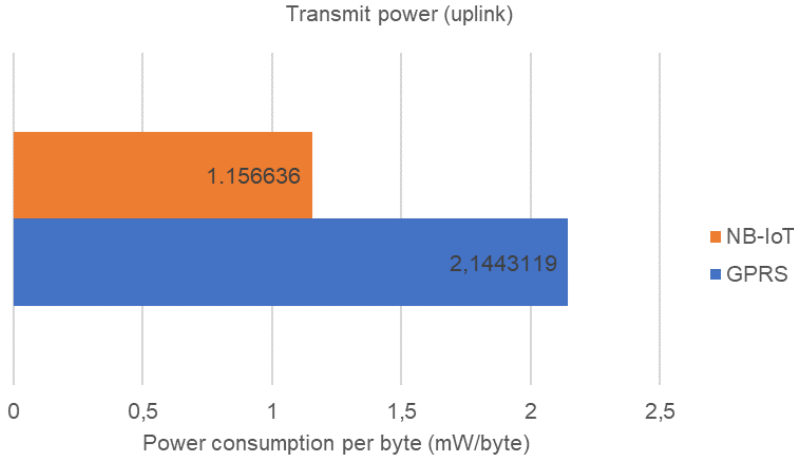

Figure 2. Uplink Transmit Power (Smaller = Better)

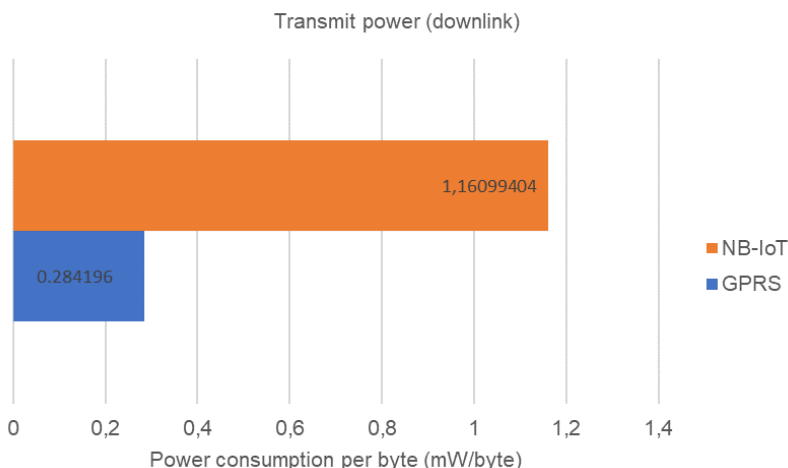

Figure 3. Downlink Trasnmit Power (Smaller = Better)

Figure 4 shows estimated common 18650 Li-lon battery life with capacity $2560 \mathrm{mAh}$ or $8.448 \mathrm{~W} / \mathrm{h}$ [31]. This estimation does not include external component other than the modem itself.

Further, the estimated battery life was calculated using formula in Equation 5. The scenario used to compute the estimated battery life was: transaction interval was every hour, data length was 512 Byte, and battery capacity was $8.448 \mathrm{~W} / \mathrm{h}$.

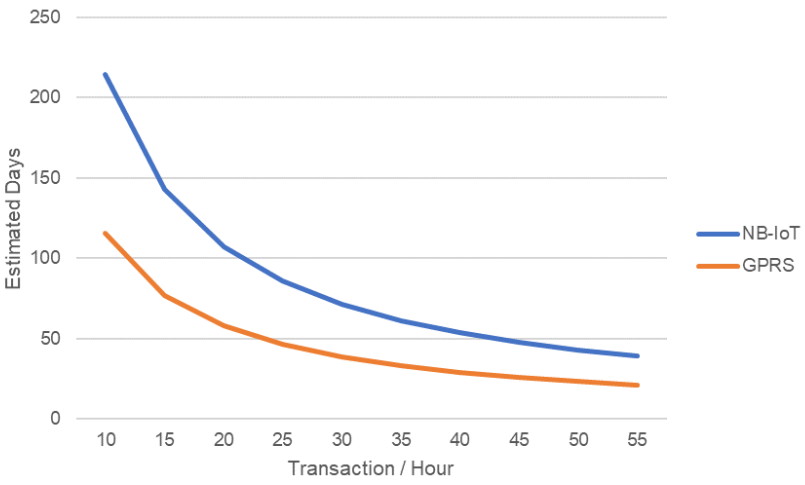

Figure 4. Estimated Battery Life

From Figure 4, we can conclude that where if the number of transactions increases, the power usage will decrease for both of GPRS and NB-loT technology. The GPRS consumed more energy more than NB-loT for the same number of transactions, which means that NB-IoT is better than GPRS in the aspect of energy savings.

\section{Conclusion}

IoT data can be sent using GPRS and NB-loT technology. This paper compared the GPRS and the NB-loT technology for sending loT data using TCP and UDP protocols. The coverage area used in the deployment was Bandung, Indonesia. From the results, we can conclude that throughput, packet loss, and energy usage are superior to the NB-IoT network compared to GPRS. This result means that NB-IoT is more feasible to send loT data better than GPRS.

Cite: Bima, I., Suryani, V., \& Wardana, A. (2020). A Performance Analysis of General Packet Radio Service (GPRS) and Narrowband Internet of Things (NB-loT) in Indonesia. Kinetik: Game Technology, Information System, Computer Network, Computing, Electronics, and Control, 5(1). doi:https://doi.org/10.22219/kinetik.v5i1.947 
Research development can be done by utilizing both technologies for real loT applications, for example, smart tracking, smart cities, or smart transportation. Thus, the performance of both technologies can be seen better when they are used in real applications.

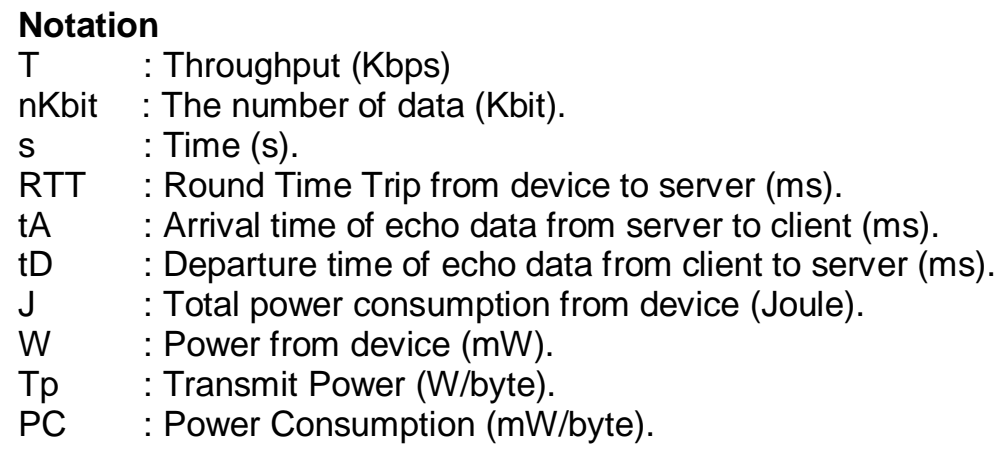

\section{Acknowledgement}

Thank you to the Internet of Things Studio, Telkom University which has become a place for researchers to develop this journal research. Special thanks to Telkomsel, Mr. Ketut Susila, and Yanuar Adhinagara for trial SIM card and NB-loT network access. Hopefully, this research can make a major contribution to the advancement of technology in Indonesia.

\section{References}

[1] D. A. K. Karimi, "What the Internet of Things (IoT) Needs to Become a Reality," Free. White Pap., Pp. 16, 2013.

[2] A. A. Wardana, A. Rakhmatsyah, A. E. Minarno, and D. R. Anbiya, "Internet of Things Platform for Manage Multiple Message Queu ing Telemetry Transport Broker Server," Kinet. Game Technol. Inf. Syst. Comput. Network, Comput. Electron. Control, Vol. 4, No. 3, Pp. 197-206, 2019. https://doi.org/10.22219/kinetik.v4i3.841

[3] S. Vashi, J. Ram, J. Modi, S. Verma, and C. Prakash, "Internet of Things (IoT): A vision, architectural elements, and security issues," Proc. Int. Conf. IoT Soc. Mobile, Anal. Cloud, I-SMAC 2017, no. February 2017, Pp. 492-496, 2017. https://doi.org/10.1109/I-SMAC.2017.8058399

[4] V. Suryani, S. Sulistyo, and W. Widyawan, "Internet of Things (IoT) Framework for Granting Trust among Objects," J. Inf. Process. Syst., Vol. 13, No. 6, Pp. 1613-1627, 2017. https://doi.org/10.3745/JIPS.03.0088

[5] J. Xu, J. Yao, L. Wang, Z. Ming, K. Wu, and L. Chen, "Narrowband internet of things: Evolutions, technologies, and open issues," IEEE Internet Things J., Vol. 5, No. 3, Pp. 1449-1462, 2018. https://doi.org/10.1109/JIOT.2017.2783374

[6] A. D. Zayas and P. Merino, "The 3GPP NB-IoT system architecture for the Internet of Things," 2017 IEEE Int. Conf. Commun. Work. ICC Work. 2017, Pp. 277-282, 2017. https://doi.org/10.1109/ICCW.2017.7962670

[7] M. Pennacchioni, M. G. Di Benedette, T. Pecorella, C. Carlini, and P. Obino, "NB-loT system deployment for smart metering: Evaluation of coverage and capacity performances," 2017 AEIT Int. Annu. Conf. Infrastructures Energy ICT Oppor. Foster. Innov. AEIT 2017, Vol. 2017Janua, No. 1, Pp. 1-6, 2017. https://doi.org/10.23919/AEIT.2017.8240561

[8] M. Zeinali, J. Thompson, C. Khirallah, and N. Gupta, "Evolution of home energy management and smart metering communications towards 5G," Proc. 2017 8th Int. Conf. Netw. Futur. NOF 2017, Vol. 2018-Janua, Pp. 85-90, 2018. https://doi.org/10.1109/NOF.2017.8251225

[9] A. R. Kulkarni, M. S. Ballal, and S. S. Rajurkar, "Developing SMARTGRID Projects with Global Perspective in Indian Scenario," vol. 487, Springer Singapore, 2018, Pp. 19-28. https://doi.org/10.1007/978-981-10-8249-8_1

[10] S. Persia and L. Rea, "Next generation M2M Cellular Networks: LTE-MTC and NB-loT capacity analysis for Smart Grids applications," AEIT 2016 - Int. Annu. Conf. Sustain. Dev. Mediterr. Area, Energy ICT Networks Futur., 2016. https://doi.org/10.23919/AEIT.2016.7892789

[11] S. Persia, C. Carciofi, and M. Faccioli, "NB-IoT and LoRA connectivity analysis for M2M/loT smart grids applications," 2017 AEIT Int. Annu. Conf. Infrastructures Energy ICT Oppor. Foster. Innov. AEIT 2017, Vol. 2017-Janua, Pp. 1-6, 2017. https://doi.org/10.23919/AEIT.2017.8240558

[12] J. Shi, L. Jin, J. Li, and Z. Fang, "A smart parking system based on NB-loT and third-party payment platform," 2017 17th Int. Symp. Commun. Inf. Technol. Isc. 2017, Vol. 2018-Janua, Pp. 1-5, 2018. https://doi.org/10.1109/ISCIT.2017.8261235

[13] W. Zhai, "Design of narrowband-IoT oriented wireless sensor network in urban smart parking," Int. J. Online Eng., Vol. 13, No. 12, Pp. 116126, 2017. https://doi.org/10.3991/ijoe.v13i12.7886

[14] Y. Zhu, G. Jia, G. Han, Z. Zhou, and M. Guizani, "An NB-loT-based smart trash can system for improved health in smart cities," 201915 th Int. Wirel. Commun. Mob. Comput. Conf., Pp. 763-768, 2019. https://doi.org/10.1109/IWCMC.2019.8766748

[15] S. Chen, C. Yang, J. Li, and F. R. Yu, "Full Lifecycle Infrastructure Management System for Smart Cities: A Narrow Band loT-Based Platform," IEEE Internet Things J., Vol. PP, No. C, Pp. 1-1, 2019. https://doi.org/10.1109/JIOT.2019.2923810

[16] S. Duangsuwan, A. Takarn, and P. Jamjareegulgarn, "A Development on Air Pollution Detection Sensors based on NB-loT Network for Smart Cities," Isc. 2018 - 18th Int. Symp. Commun. Inf. Technol., No. Iscit, Pp. 313-317, 2018. https://doi.org/10.1109/ISCIT.2018.8587978

[17] S. Duangsuwan, A. Takarn, R. Nujankaew, and P. Jamjareegulgarn, "A Study of Air Pollution Smart Sensors LPWAN via NB-IoT for Thailand Smart Cities 4.0," 2018 10th Int. Conf. Knowl. Smart Technol. Cybern. Next Decad. KST 2018, Pp. 206-209, 2018. https://doi.org/10.1109/KST.2018.8426195

[18] J. Wang, J. Su, and R. Hua, "Design of a Smart Independent Smoke Sense System Based on NB-loT Technology," Proc. - 2019 Int. Conf. Intell. Transp. Big Data Smart City, ICITBS 2019, Pp. 397-400, 2019. https://doi.org/10.1109/ICITBS.2019.00104

[19] K. Mikhaylov et al., "Multi-RAT LPWAN in Smart Cities: Trial of LoRaWAN and NB-loT Integration," IEEE Int. Conf. Commun., Vol. 2018-May, 2018. https://doi.org/10.1109/ICC.2018.8422979

[20] P. Jorke, R. Falkenberg, and C. Wietfeld, "Power Consumption Analysis of NB-loT and eMTC in Challenging Smart City Environments," 2018 IEEE Globecom Work. GC Wkshps 2018 - Proc., 2019. https://doi.org/10.1109/GLOCOMW.2018.8644481 
[21] M. El Soussi, P. Zand, F. Pasveer, and G. Dolmans, "Evaluating the Performance of eMTC and NB-IoT for Smart City Applications," IEEE Int. Conf. Commun., Vol. 2018-May, Pp. 1-7, 2018. https://doi.org/10.1109/ICC.2018.8422799

[22] A. Gerber, Z. M. Mao, and O. Spatscheck, "A Close Examination of Performance and Power Characteristics of 4G LTE Networks Categories and Subject Descriptors," MobiSys '12 Proc. 10th Int. Conf. Mob. Syst. Appl. Serv., Pp. 225-238, 2012.

[23] M. Lauridsen, R. Krigslund, M. Rohr, and G. Madueno, "An Empirical NB-loT Power Consumption Model for Battery Lifetime Estimation," IEEE Veh. Technol. Conf., Vol. 2018-June, Pp. 1-5, 2018. https://doi.org/10.1109/VTCSpring.2018.8417653

[24] B. Vejlgaard, M. Lauridsen, H. Nguyen, I. Z. Kovacs, P. Mogensen, and M. Sorensen, "Coverage and Capacity Analysis of Sigfox, LoRa, GPRS, and NB-IoT," IEEE Veh. Technol. Conf., Vol. 2017-June, 2017. https://doi.org/10.1109/VTCSpring.2017.8108666

[25] M. Lauridsen, I. Z. Kovács, P. Mogensen, M. Sørensen, and S. Holst, "Coverage and capacity analysis of LTE-M and NB-loT in a rural area," IEEE Veh. Technol. Conf., Vol. 20, Pp. 2-6, 2017. https://doi.org/10.1109/VTCFall.2016.7880946

[26] L. Benetazzo, M. Bertocco, C. Narduzzi, and R. Tittoto, "Analysis and measurement of TCP/IP performance over GPRS networks," Lect. Notes Comput. Sci. (including Subser. Lect. Notes Artif. Intell. Lect. Notes Bioinformatics), Vol. 2775, Pp. 261-275, 2003. https://doi.org/10.1007/9783-540-39867-7 28

[27] M. Chen, Y. Miao, Y. Hao, and K. Hwang, "Narrow Band Internet of Things," IEEE Access, Vol. 5, No. C, Pp. $20557-20577,2017$. https://doi.org/10.1109/ACCESS.2017.2751586

[28] P. Svoboda, F. Ricciato, W. Keim, and M. Rupp, "with and without Caching raefore , i winlpth senetwork Begain analysisfor over-I comparison calls .W Ffller- te I ontoto," 2007. https://doi.org/10.1109/WOWMOM.2007.4351701

[29] C. Yu, L. Yu, Y. Wu, Y. He, and Q. Lu, "Uplink scheduling and link adaptation for narrowband internet of things systems," IEEE Access, Vol. 5 , Pp. 1724-1734, 2017. https://doi.org/10.1109/ACCESS.2017.2664418

[30] SIMCom, T. Hongjun, and L. Ya, SIMCom7000 Hardware Design.

[31] Samsung SDI Co. Ltd, "INR18650-25R Testing and Specifications," 2013.

Cite: Bima, I., Suryani, V., \& Wardana, A. (2020). A Performance Analysis of General Packet Radio Service (GPRS) and Narrowband Internet of Things (NB-loT) in Indonesia. Kinetik: Game Technology, Information System, Computer Network, Computing, Electronics, and Control, 5(1). doi:https://doi.org/10.22219/kinetik.v5i1.947 
